\title{
Effects of Long Distance Cycling in a Hot Ambient Temperature on White Blood Cell Count
}

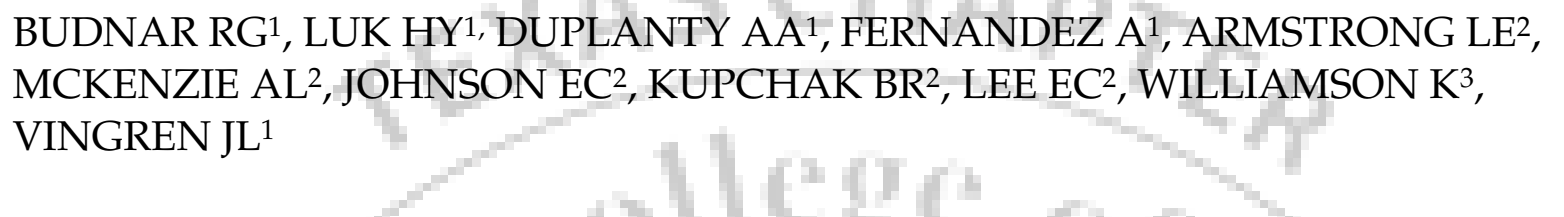

${ }^{1}$ University of North Texas, Denton, TX. ${ }^{2}$ University of Connecticut, Storrs, CT. ${ }^{3}$ Midwestern University, Wichita Falls, TX.

Category: Doctoral
Advisor / Mentor: Vingren, Jakob L., Ph.D., FACSM (Jakob.vingren@unt.edu)

Advisor / Mentor: Vingren, Jakob L., Ph.D., FACSM (Jakob.vingren@unt.edu)

\section{ABSTRACT}

Ultra-endurance events under extreme heat conditions have become popular. Prolonged exercise imposes a transient physiological stress on the immune system; this stress is augmented when exercising in hot ambient temperature conditions. To date, no field study has investigated the immune response to ultraendurance cycling under extreme heat conditions. To determine the effect of completing a $164 \mathrm{~km}$ cycling event in a hot ambient temperature on white blood cell count. Thirty-nine experienced cyclists (36 men and 3 women; $49.4 \pm 8.6 \mathrm{yr}, 83.0 \pm 15.0 \mathrm{~kg}, 176.2 \pm 7.3 \mathrm{~cm}, 19.9 \pm 6.6 \%$ body fat) participating in the August 2013 Hotter'N Hell Hundred ride held in Wichita Falls, TX, were recruited. Blood samples were collected within 2 hours of the start (PRE; 0500-0700h) and immediately after completion (POST) of the event. During the event there was minimal cloud cover and the wet bulb globe temperature was $31.4 \pm 3.8 \square \mathrm{C}$ with a maximum of $36.1 \square$ C. Samples were analyzed for complete blood count with partial differential. To examine the effect of (a) duration of heat exposure and (b) change in plasma volume, participants were grouped and compared based on finishing time (fastest and slowest 25th percentile) as well as on plasma volume (PV) change ( $>10,1-10$, and $<0 \%$ ). Total white blood cell (PRE: $5.54 \pm 0.23$; POST; $12.12 \pm 0.46 \times 109$ $\mathrm{L}^{-1}$ ), lymphocyte (PRE: $2.47 \pm 0.1$; POST: $3.52 \pm 0.22 \times 109 \mathrm{~L}^{-1}$ ), mid-size range cells (MID) (PRE: $1.27 \pm 0.08$; POST: $1.49 \pm 0.1 \times 109 \mathrm{~L}^{-1}$ ), and granulocyte (PRE: $1.81 \pm 0.11$; POST: $7.11 \pm 0.41 \times 109 \mathrm{~L}^{-1}$ ) increased significantly $(\mathrm{p}<0.05)$ from PRE to POST. This change resulted in a significant decrease in \%lymphocytes (PRE: $44.63 \pm 1.19$; POST: $29.45 \pm 1.62 \%$ ) and \%MID (PRE: $23.41 \pm 0.79$; POST: $12.89 \pm 0.86 \%$ ) and a significant increase in \%granulocytes (PRE: $31.96 \pm 1.02$; POST: $57.66 \pm 1.99 \%$ ) in circulation. No differences were observed for leukocyte subsets between the fastest and slowest 25th percentile finishers or among groups based on PV change. Prolonged cycling $(164 \mathrm{~km})$ in a hot environment induced changes in abundance of the major circulating immune cell populations; these changes were independent of exercise duration/intensity or PV change.

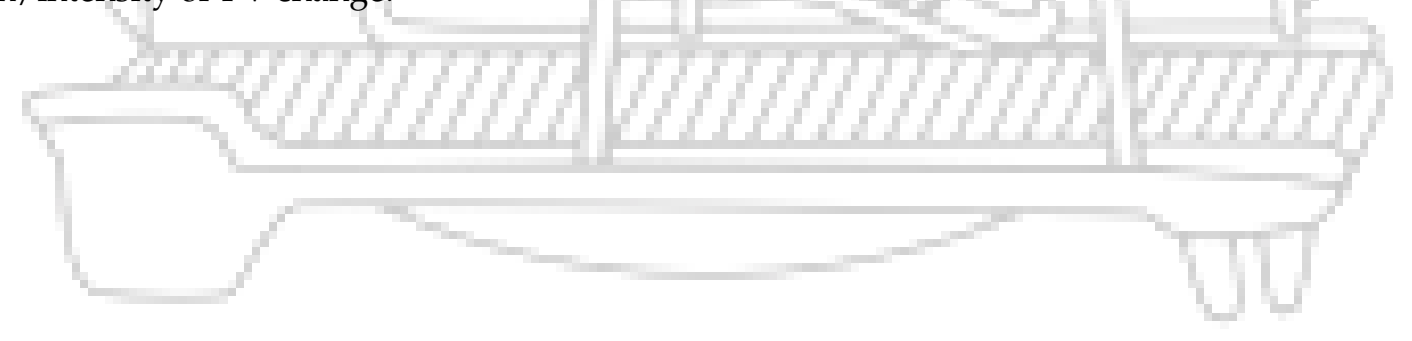

